\title{
Uncertainties when Applying the Mental Capacity Act in Dementia Research: A Call for Researcher Experiences
}

The Mental Capacity Act (MCA) has been in force in England and Wales for over a decade. Although often considered in relation to health and social care, the MCA also governs the inclusion of people with cognitive impairment in research. This includes procedures for establishing whether prospective participants are able to consent to participation. Despite its importance in research, there is little literature detailing accounts of researcher's experiences of working with the MCA's decision-making capacity procedures when conducting research. To address this deficit, we present our experiences when conducting three separate studies involving people with dementia and therefore falling within the MCA's research provisions. Study A (data collected between September 2016 and April 2017) discusses the subjectivity of capacity assessment; Study B (data collected between July and November 2017) details the negotiation of different opinions on capacity; and Study C (data collected between January and October 2013) considers tensions between legality and the principle of non-maleficence. Each of these experiences indicates a problematic degree of uncertainty within the MCA's decision-making capacity provisions. We suggest two solutions. First, more detailed guidance is required, responding to uncertainties emerging in practice. Second, researchers should be encouraged to publish their experiences of working with the MCA. In outlining our own experiences, we hope to initiate such a body of work.

Keywords: dementia, research, capacity, consent, ethics

\section{Introduction}

The Mental Capacity Act 2005 (MCA) (Mental Capacity Act 2005) has been in force in England and Wales for over a decade. The MCA is a major piece of legislation which aims to support and safeguard adults who are considered potentially vulnerable to exploitation when making decisions. The principles and procedures within the MCA therefore have implications for health and social care processes, people with cognitive impairment and their families, and research practice and governance. Though initially 
broadly welcomed, the MCA has stirred some controversy during its brief history, particularly in relation to the health and social care sectors (Law Commission 2017; Sheather 2006). However, problems are not limited to health and social care; researchers also experience challenges when working within the parameters of the MCA (Parker, Penhale, and Stanley 2011). Despite this, there is a limited body of knowledge detailing researchers' experiences of working with the MCA when conducting projects, and therefore relatively little advice on how to navigate challenges as they arise. To address this issue, this paper outlines the experiences of three doctoral researchers when using the MCA in their work.

The original aims and current controversies of the MCA are first introduced. The research guidelines within the MCA are then outlined. A perceived general lack of understanding of the Act's research-specific provisions is considered, followed by an observation that there is a lack of literature detailing researchers' experiences of the MCA in practice. It is argued that this deficit should be rectified for two reasons: to stimulate debates that can lead to improvement, and to provide guidance for future researchers. Then, examples of challenges faced by three researchers when using the MCA in their respective research projects are described. Considered together, these three examples highlight a significant issue of uncertainty when operationalising the MCA research guidelines during fieldwork. In conclusion, it is argued that the development of a collection of resources outlining researcher experiences, providing examples of successes and challenges in practice, would go some way to addressing the problem of uncertainty.

\section{The Mental Capacity Act and Research Guidelines}

The MCA has been in force since 2007. It legislates on the decision-making process for people aged 16 years and older living in England and Wales who have 'an impairment 
of, or disturbance in, the functioning of the mind or brain' (Mental Capacity Act 2005). The MCA is relevant to people with dementia as a condition that impairs cognition, although people without a clinical diagnosis of a condition may also be supported to make decisions through the processes of the Act. The MCA separates people with cognitive impairments into two categories: those considered to have the legal capacity to make decisions, and those considered to lack such capacity. Legislators intended this legal distinction to prevent the exclusion and exploitation of people with cognitive impairments when making decisions about themselves. Thus, the MCA seeks to support decision-making among people with cognitive impairments who have legal capacity and safeguard those who lack it, while still encouraging their participation in decisions where possible. This is in response to historic reliance on proxy decision-making (Brown, Barber, and Martin 2009).

The MCA has stirred debate since its inception. Notably, in actively discriminating legal statuses based upon impairment the Act seemingly contravenes Article 12 of the United Nations Convention on the Rights of Persons with Disabilities (UNCRPD), which protects 'equal recognition before the law', and which the United Kingdom (UK) ratified in 2009 (Bartlett 2012). More recently, the MCA's Deprivation of Liberty Safeguards (DoLS), a later addition to the Act, legislating when people can be detained against their will (Mackenzie and Watts 2010), have drawn criticism (House of Lords 2014; Law Commission 2017). A House of Lords Select Committee review was established in 2013. It found that implementation had failed to satisfy expectations because the MCA was poorly understood and often viewed as optional guidance as opposed to mandatory legislation (House of Lords 2014). In line with these findings, studies of affected professions have discovered varied understanding of the MCA among staff, though most practice is still within the MCA guidelines, albeit unwittingly 
(Manthorpe, Rapaport, and Stanley 2008; Manthorpe et al. 2011; Wilson, Seymour, and Perkins 2010). Amongst such substantial debates, problems regarding the MCA's research-specific guidelines have received relatively little attention.

Part 1, Sections 30-34 of the MCA outline the guidelines for conducting research with people with cognitive impairments. These sections outline several requirements for research involving people who have 'an impairment of, or disturbance in, the functioning of the mind or brain'. Such research must be approved by an appropriate ethics committee, as specified by the Act, and must focus on the impairment in question. To include participants who lack capacity, it must be shown that the research would be less effective without their participation. Research must also either benefit the participant directly, or improve knowledge of causes, treatments or care relating to the condition in question, while not unduly burdening the participant.

The most significant implication for research practices following the implementation of the MCA has been the requirement to assess the decision-making capacity of potential participants to gain their consent to be in the project. An assessment is conducted to distinguish would-be participants who possess the capacity to consent to participate from those who are determined as lacking that capacity. The assessment is a two-stage functional test of capacity consisting of two questions: is there an impairment in the person's mind or brain that affects how it works (not necessarily a diagnosis of a condition); and if so, does the impairment stop the person being able to make the particular decision at the time it needs to be made? To answer this second question, the assessment contains four criteria which the person should demonstrate they can fulfil. These are: to understand information about the decision; to retain that information for long enough to use it; to weigh up the information and understand the consequences of their decision; and to communicate their decision somehow. A person 
must demonstrate all four of these criteria to be considered to possess decision-making capacity. Individuals with capacity may then legally consent, if they wish, to participate in the research. For those assessed as lacking capacity to consent, procedures necessitate the researcher to liaise with certain third parties known as Personal Consultees and Nominated Consultees. These individuals provide their perspectives on whether the person assessed as lacking capacity would or would not want to participate in the research, if he or she could provide their own consent (Dobson 2008).

\section{Lack of Researcher Experiences}

Throughout its brief history, the principle areas of use, and therefore discussion, of the MCA have been in decisions relating to healthcare, social care and finances. As a result, there is relatively little understanding and dialogue pertaining to the MCA's researchspecific provisions (Parker, Penhale, and Stanley 2011). The primary argument of this paper is that a greater body of material charting the experiences of researchers who have used the MCA could aid researchers. We believe that this could: 1) promote discussions on the challenges of the MCA in research and 2) provide much-needed practical guidance for researchers. Ultimately, improvement of the research experience cannot occur without the identification of challenges and learnings.

First, greater discussion is required as a means of highlighting the challenges currently being experienced by researchers in practice. During the time that the MCA has been in operation, issues have been raised concerning various aspects of the Act, for example concerning DoLS (House of Lords 2014; Law Commission 2017) and the UNCRPD (Bartlett 2012), as noted above. While the majority of these issues have been considered in the context of health and social care, research is not immune from concerns. It is unlikely that legislators can improve the MCA without the development of a significant evidence-base demonstrating the performance of the Act in practice. 
Beyond this, discussion stemming from the aggregation of such material may in itself suggest reasonable responses to recurring issues, potentially driving necessary improvements. There also needs to be opportunities for researchers to discuss their positive examples of the MCA in practice, to enable learning to be shared.

Second, greater guidance could assist researchers whose work falls under the MCA, particularly those engaging with the guidelines for the first time. Our personal communications with fellow researchers indicate that adhering to MCA guidance can be a challenging experience. The Act bestows upon researchers a substantial responsibility. This responsibility necessitates corresponding guidance and support. While resources on the correct implementation of the MCA research guidelines are useful (e.g. Department for Constitutional Affairs 2007; Dobson 2008; National Mental Capacity Forum 2017), learnings that could be taken from researchers' real-world examples are missing. A body of literature outlining the experiences of researchers when working under the MCA could somewhat address this deficit, sharing learnings on what has worked well and highlighting what procedures have been successful.

For these two reasons, we believe that a body of literature detailing researchers' experiences of using the MCA when conducting research could benefit the research community. This paper does not solely constitute a call to develop a body of literature recounting researcher experiences; we also intend to spearhead this movement by sharing our accounts of implementing the MCA guidelines when conducting our doctoral research projects with people with dementia. These experiences are then collectively considered to highlight one problem when implementing the MCA in research - that of uncertainty. 


\section{Our Experiences}

\section{Study A - Subjectivity of Assessment}

Study A was a doctoral research project exploring perceptions and experiences of informal care from the perspectives of people with dementia and their care networks. The study recruited seven people with dementia living in their own home or that of a relative in three Midlands counties in the UK. Participants had early to moderate stages of dementias including Alzheimer's disease, vascular dementia, mixed dementia and fronto-temporal dementia. Six participants were male, and ages ranged from 74 to 92 years old. Semi-structured interviews were initially conducted with participants with dementia. During this interview, care networks were eco-mapped (see Rempel, Neufeld and Kushner 2007) to generate a visual representation of the key actors in care from the perspective of the person with dementia. Identified carers were subsequently interviewed individually, before a final follow up interview was conducted with the person with dementia.

During this study, the researcher was required to conduct capacity to consent assessments with potential participants. One such assessment was undertaken with $\mathrm{Mr}$ Hargreaves, ${ }^{1}$ who had been living with Alzheimer's disease for four and a half years. He was the first person with dementia recruited into the study and was the researcher's first experience of a capacity to consent assessment. Mr Hargreaves had difficulty satisfying the assessment requirements. The four requirements of decision-making capacity are: a) to understand the information relevant to the decision; b) to retain that information; c) to use or weigh that information as part of the process of making the decision; and d) to communicate his decision (whether by talking, using sign language

\footnotetext{
${ }^{1}$ All participant names are pseudonyms
} 
or any other means). For a person to have capacity, they must satisfy each of these criteria. Mr Hargreaves was able to retain information and communicate a decision, and seemed to have some understanding. However, he did not evidence an ability to weigh information within a decision-making process, appearing to reiterate rather than assess information. As a result, Mr Hargreaves narrowly lacked capacity. That is, the researcher was slightly unconvinced of Mr Hargreaves having satisfied a personal conception of sufficient capacity. However, the MCA allows for a second attempt at the assessment. The second time, the researcher was convinced that Mr Hargreaves had decision-making capacity and thus progressed to the consent procedure. This time, $\mathrm{Mr}$ Hargreaves appeared to weigh the information and make a more obvious judgement, making an appeal to the potential usefulness of his involvement. The researcher deemed this to be evidence of an assessment of the information in terms of pros and cons. In another similar case, the researcher deemed Mr Smith, a 75 year-old who had been living with fronto-temporal dementia for ten years, to lack capacity because he appeared to be repeating information rather than weighing it up. This is difficult to judge, but the researcher based his decision on Mr Smith's consistent verbatim repetition. The example of Mr Hargreaves raises questions regarding subjectivity. His position on the border between having and not having capacity in terms of the researcher's interpretation of the MCA guidance highlights a substantial element of subjectivity. As a researcher, one must judge a person's responses against one's own interpretation of capacity (Banner 2012), because although the MCA offers guidance on the meaning of capacity, as a concept it cannot be objectively measured, discussed further below. While this allows flexibility, it can be concerning given the gravity of the judgment that is being made on the person. Out of a group of several assessors, it is probable that some would have deemed Mr Hargreaves to lack capacity, while some would have judged 
him to have capacity. This probable variation is caused by an inherent subjectivity in the practical implementation of the MCA's research provisions regarding capacity assessment by a researcher.

The MCA aims to reduce subjectivity by stringently defining the assessment process in terms of the composite requirements of capacity. This is successful to an extent, because it narrows the range of probable interpretations. However, no matter the minutiae of the MCA or the quality of the deliberation underpinning it, subjectivity will remain. This is because the central concepts within this issue are indefinite and those concepts must be enacted by a human researcher. There is no absolute level of capacity that is required for making a given decision, and even if there was, there would likely be no unquestionable method for assessing that capacity in practice. ${ }^{2}$ Banner (2012) has noted similar issues of normative judgement regarding the assessment of mental capacity in clinical practice. The concepts of mental capacity legislation therefore open up a space in which subjectivity is unavoidable when its principles are implemented in real-life situations. This places people like Mr Hargreaves in a situation in which their rights are somewhat dependent upon the subjectivities of a third party. This could be harmful for the people being assessed because they may have legal rights retracted based upon fallible judgments, and it is also an intimidating realisation for assessors who are expected to exercise great discretionary power over other people. As a result,

\footnotetext{
${ }^{2} \mathrm{~A}$ foundational principle of the MCA is that an assessor must assume capacity unless there is evidence to the contrary. Therefore, in cases in which there is uncertainty, one could argue that assessors should conclude that the person being assessed does have capacity. Such an argument is open to interpretation, further evidencing the influence of subjectivity within the application of the MCA's provisions.
} 
subjectivity can be considered a substantial issue when implementing the MCA's research provisions in practice.

The indefinite nature of core concepts means that capacity is partially a matter of interpretation in assessment. The purpose of this critique is not to argue that this subjective aspect is a fault of the MCA, for it is not. Instead, we are arguing that the concept of capacity defies sufficient definition and hence allows for a wide range of different interpretations. It is improbable that a completely objective concept of capacity, satisfying traditional notions of validity and reliability, could ever be devised. However, while capacity assessment cannot be absolutely objective, it can likely be more so than it currently is. The concepts upon which the MCA draws might feasibly be further specified as a means of limiting potential interpretations. The assessment process could benefit from greater attention to the meaning and, more importantly, the operationalisation of concepts such as understanding and capacity. A useful future addition to the MCA might feasibly stem from the assembly of a panel of people with cognitive impairments, lawyers, clinicians, carers, cognitive scientists, social scientists and philosophers, dedicated to developing more sophisticated definitions of key concepts and comprehensive practical guidance regarding their use.

\section{Study B - Differences in Interpretation of Capacity}

Study B explored the decision-making practices relating to which belongings people with dementia take with them when moving into care homes. It also explored the perception of risk and how this manifests in decision-making relating to the opportunities people with dementia have to use functional objects in daily life. The project took place in a local authority residential care home in Hampshire, UK. A total of 40 participants took part, consisting of 15 people with a mild to severe dementia, 17 care home staff and 8 family members who visited relatives in the home. 
Ethical approval was granted by the Social Care Research Ethics Committee, a Health Research Authority committee, to include people with dementia on the basis they would be split into two groups. One group would include people assessed as having capacity to consent: they would be invited to take part in interview, participant observations, and documentary research (Coffey and Atkinson 1996). Another group would include people assessed as lacking capacity to consent. This group would be excluded from taking part in interviews; however, should a Personal or Nominated Consultee give the opinion they believed the person would like to take part, they were included in participant observations and documentary research.

The registered manager of the care home was recruited to act as a gatekeeper. One of the roles of the gatekeeper was to inform the researcher of those she believed had capacity to consent and those who she believed did not. This was to ensure the accurate distribution of participant information sheets. Whilst there were many minor challenges identified in this process, the most significant was the differences in interpretation of capacity to consent.

To begin with, the researcher spent one month in the care home conducting a 'hanging out' period. This is a crucial part of ethnographic work in which time is spent in the natural environment prior to data collection (Bernard 1995). The 'hanging out' period resulted in three positive consequences. First, it enabled interactions with all people with dementia, staff and family members, without focusing on a research question. Second, it enabled people with dementia, staff and family members to ask the researcher questions. Third, it helped the researcher get an idea of who may or may not pass the capacity to consent assessment.

Whilst 'hanging out', there were times when the subject of capacity to consent was discussed between care staff and the researcher. It was then that the researcher 
began to identify variations in care staff's understandings of capacity. Some staff openly stated they did not know whether people had capacity to consent or not; other staff tended to presume a lack of capacity. This presumption was often linked to an 'established assessment', which was a DoLS assessment. Whilst legally it is clear that DoLS is granted for the purpose of either permitting or denying a person freedom to leave the care home, it appeared in practice to confirm the widely held belief that the person with dementia lacked capacity more generally. Conversely, care staff would also suggest a person to be a 'good participant'. This was often qualified by the fact the person was a 'good talker' or that they had 'lots of belongings' resulting in their appropriateness for the research project. There were no obvious signs of a logical or thoughtful decision-making process in relation to the assessment of mental capacity.

Differences in interpretation of capacity to consent were also found in the list of potential participants compiled by the gatekeeper. Following the 'hanging out' period, the researcher had formed ideas of who she perceived might pass the capacity to consent assessment and had also shown an interest in the research. The list compiled by the gatekeeper placed the majority of people with dementia as lacking capacity to consent and therefore requiring a Personal or Nominated Consultee. This did not fit with the researcher's interactions, resulting in a dilemma. If the researcher questioned the gatekeeper's assessment of capacity, this could have implications. It could be viewed that the researcher was attempting to influence or coerce participation in the recruitment process. It could also disrupt the developing research relationship. If the researcher failed to question the identified discrepancies, this could result in people with dementia being excluded, at best from the interview section of the research, and at worst the entire project, thus denying them the opportunity to take part in decision-making and opportunities to participate. 
A decision was made to question the gatekeeper's assessment of capacity. This proved worthwhile, as it allowed both the researcher and the gatekeeper to discuss their interpretations, challenging how some people with dementia were negatively positioned. Whilst it was agreed that some people with dementia were likely to fail the capacity to consent assessment, other cases were not so clear. It was, however, clear that the gatekeeper and the researcher were coming from opposing standpoints. The gatekeeper was erring on the side of caution; that if capacity was questionable they were likely to fail and therefore a Consultee should be appointed. In opposition, the researcher was erring on the side of least restriction until capacity to consent was formally established. This also enabled the researcher to conduct the capacity to consent assessment in line with the MCA's intended aims; to support decision-making, safeguard those assessed as lacking capacity, and encourage participation where possible.

Whilst the capacity to consent assessment appears straightforward, the context within which it is applied is less so. Of course, legislation can never pre-empt the minutiae of all potential contexts. However, the issue of contextual complexity could be moderated through our suggested development of a literature outlining researcher experiences. This project exemplifies that interpretation of the MCA can vary, leading to confusion over who can and cannot take part in research. The Act therefore introduces an additional complexity and potential obstacle to important research relationships between researchers and gatekeepers. The Act allows for the consultation of others to assist the assessment process, but it expressly forbids the prejudgement of capacity by any third party, regardless of their relationship with the person being assessed. From care staff suggesting 'good participants' based on them having 'lots of belongings' or being a 'good talker', to differences in interpretation between the researcher and registered manager relating to mental capacity, there were clear 
variations in knowledge and understanding. However, working with the registered manager, discussing the differences in interpretation, enabled shared decision-making to take place. Two recommendations for future care home studies including people with a dementia would be (1) to ensure that mental capacity tests are conducted in collaboration with the registered manager, and (2) to provide an information session to all care staff outlining the research methods and how capacity will be assessed. These two measures may ensure that potential participants are given every opportunity to take part, and that the joint assessment carries reliability, and that all staff are well informed of what constitutes mental capacity for research purposes.

\section{Study C-Balancing Legality and Non-Maleficence}

Study C explored the use of Assistive Technologies to aid the performance of Activities of Daily Living in two locations: the person with dementia's own home and care homes. One data collection method within the project consisted of mapping relevant data from the social care records of care home residents with dementia. Access to care records necessitated capacity to consent assessments to be conducted with 38 eligible care home residents with dementia. Some of these residents were in a severe stage of dementia at the time of the assessment; they appeared bewildered by their surroundings or were unresponsive at all times. The researcher, entering the bedroom of a person who was clearly non-responsive and unable to leave their bed independently, was struck by a dilemma regarding the appropriate next action to take.

The dilemma arose because while the individual was eligible to participate in the project and therefore to have a capacity to consent assessment, the ethical principle of non-maleficence, to do no harm, was never more so apparent than for these individuals. The researcher wondered whether the act of undertaking capacity assessment would be distressing for people with a severe state of dementia. For example, one woman cried 
when asked questions, suggesting harm, yet questioning is a legal requirement. Four options seemed available to the researcher, each with distinct legal and moral implications. The first option was to run through the capacity to consent assessment, with as noted above the risk that the person with dementia might become distressed by a stranger in his or her bedroom, leaning over their bed and talking to them. The second option was to not conduct the assessment, but instead indicate on the assessment record that the person was assumed to lack capacity and contact their Personal Consultee immediately. However, this option would clearly contravene the first principle of the MCA: to assume capacity unless shown otherwise (Mental Capacity Act 2005), and the researcher was unsure if this would be an illegal act.

This issue emerges from tensions between practically legislating for people who are clearly unresponsive and respecting the ideal that nobody can be assumed to lack capacity. This ideological tension is beyond the scope of this paper. While some may argue that there should be no requirement to conduct an assessment in such scenarios, this contravenes the MCA's key premise, and therefore requires a considerable amount of further debate. Given that the researcher was alone ${ }^{3}$ with the person with dementia, the third option was to pretend that the assessment had been conducted. The record would show that the person lacked capacity to consent and the Personal Consultee would be contacted. Whilst this appeared the easiest option, it was discarded immediately by the researcher as being immoral, unethical, and possibly illegal. The fourth option was to exclude the person from the study immediately, having not conducted an assessment, nor contacted their Personal Consultee. It was felt that this

\footnotetext{
${ }^{3}$ Staff at the care homes in question were too busy to be present during assessments, meaning that the researcher was left to conduct them alone.
} 
option would deny the person with dementia their legal right to assessment and potential participation, perpetuating the history of exclusion in research (Iphofen 2009).

The first option was chosen: to run through the capacity to consent assessment with all potential participants with severe dementia. These individuals had a right to participate in an assessment regardless of their apparent condition. Whilst the risk of distressing these individuals was salient, there were ways to reduce this risk through communication skills; for example, a soft tone of voice, non-threatening body language, and holding their hand. It was also perceived that the presence of, and interaction with, the researcher was more beneficial to the person's wellbeing compared to remaining alone in their bedroom all day.

A final challenge with conducting assessments with these individuals was that MCA guidance (Dobson 2008) states that a non-response cannot be interpreted as evidence of a lack of capacity. Whilst person-to-person it was very clear that these people did not have capacity, an issue was how to demonstrate, within the constraints of the assessment record, that the person with dementia truly lacked capacity and did not merely have impaired communication. The researcher had to be careful, as the reliability of decisions can be queried (Hotopf 2005). However, the care home staff advised that these individuals no longer experienced moments of lucidity, and thus returning for an assessment at a more appropriate time was futile. Furthermore, the researcher could not foresee a circumstance by which another assessor would find these individuals had capacity and therefore had confidence in the reliability and validity of the assessment outcome, unlike in Study A above.

It was only once the researcher began fieldwork that the seemingly clear guidelines in the MCA became murky. The unforeseen challenge arose from a mismatch between legal requirements and the research ethical principle of non- 
maleficence to do no harm. Addressing this uncertainty was dependent upon the researcher's moral judgement and assessment of the most correct action. As we discuss below, we hope to prevent such dilemmas from occurring, or at least help other researchers to know what actions they might take, by sharing our experiences here.

\section{Discussion}

It is our primary contention in this paper that the three experiences outlined above each speak to challenges stemming from uncertainty. Study A reveals uncertainties around key concepts in the MCA, resulting in an inherent subjectivity within assessment. Study B speaks of uncertainties concerning the contexts within which the MCA is enacted, potentially provoking tensions between various stakeholders, but also potentially excluding people with dementia from research. Study C evidences uncertainties regarding the negotiation of research ethical principles and legality when the two do not entirely align, as well as the distinct challenges faced when working with people with severe dementia.

The challenges experienced during our engagement with the MCA in our research projects can be viewed as issues of uncertainty. To an extent, it is inevitable that early career researchers will desire greater certainty due to insecurities bound up with their juniority. This also entails questions regarding the necessity of experience. The MCA allows any researcher, with or without experience, to conduct assessments, and some may question whether minimum experience requirements should be developed. However, we would caution against over-regulation in this area due to the potential to disincentivise the involvement of early career researchers in participatory studies on cognitive impairment. While we cannot speak for others, SCREC approved all three of our research projects despite our lack of prior experience, and only questioned our general experience of interacting with people with dementia, rather than 
focussing on capacity assessment. Indeed, for each study the procedural ethics process focussed on other aspects of the research and largely overlooked the issue of capacity assessment.

Such issues are not sufficient to dismiss issues of uncertainty altogether. Furthermore, the uncertainties raised within each study are significant: How does one solidify the borders of a concept such as capacity? How does one negotiate the complex interpersonal contexts surrounding assessment? How does one reconcile moral and legal tensions at the extremes of cognitive decline? In each situation, satisfactory answers to these pressing and unanticipated uncertainties cannot be found within the MCA's current guidance. Yet these situations are unlikely to be isolated incidents. It is therefore evident that such uncertainties need to be rectified in the interests of both researchers and research participants. There are two parts to this problem. First, uncertainties partially result from insufficient guidance, though it should be acknowledged that no guidance can remove all uncertainty. Second, experiences of these uncertainties are not available to the research community as an accessible resource for driving improvement. Regarding the first issue, addressing some uncertainties via the provision of updated guidance could be a viable route for improving the MCA's research provisions, as well as the experiences of researchers who put those provisions into practise. In the discussed cases, the complex issues involved may have a degree of inherent uncertainty. However, even if this is the case, there is likely room for improvement. Issues stemming from uncertainty can be somewhat mitigated by efforts to provide greater certainty regarding specific aspects of the MCA. In practice, this entails the careful revision of what is already in place. Each challenge stemming from the MCA, such as those arising in our own experiences, should first be recognised as a current shortcoming, and should then be addressed through further clarification of how to approach that specific 
challenge. Ultimately, this solution entails the development of more thorough guidance on the MCA, considering various potential problems in greater detail. The National Institute for Health and Care Excellence will publish further MCA guidance this year, although the draft consultation (NICE 2017) appears to continue the widespread focus on health and social care practice at the expense of research.

It is important to note that guidance on research is not solely relevant for researchers. As raised in study B, research could be aided by greater gatekeeper awareness of the MCA's research provisions. For example, awareness that assessment is decision-specific could prevent gatekeepers from prejudging who is suitable for participation. Of course, we recognise that gatekeepers such as care home managers have little incentive to familiarise themselves and their staff with the MCA's research provisions. Understandably, the onus will always fall on researchers in practice, and the most effective strategy might be to prepare researchers for gatekeeper interactions concerning capacity legislation.

The solution to the second problem, the lack of accounts of researcher's experiences when working within the MCA, is to develop a body of literature that outlines varied experiences. Such a resource should detail problems, as is the case in this paper, as well as success stories. Just as it is important to recognise difficulties, so it is also vital that good practice is shared widely. The proliferation of such a literature, chiefly concerned with improving the MCA in terms of both provision and application, could do much to improve the experiences of researchers and participants alike. With a greater awareness of the experiences of others, researchers could refine their practice. They might also benefit psychologically from an appreciation that difficulties are not unique to their experience, addressing any uncertainty stemming from their juniority. Researchers are uniquely positioned in relation to the MCA and the research process, 
and are therefore likely to recognise challenges and possible resolutions that are less immediately evident to other stakeholders.

This second point in particular raises further questions regarding the nature of uncertainty. As noted, a certain degree of uncertainty is inevitable when dealing with such complex issues. Our primary intention is that the development of further guidance and a literature base recounting researcher experiences should promote further certainty for researcher. We envisage these initiatives as limiting uncertainty where possible, rather than removing uncertainty altogether, as this latter development is practically impossible. In light of the persistence of some inevitable uncertainty, a secondary pragmatic outcome of these developments could be developing the abilities of researchers to cope with uncertainty in the field. In the complex contexts of real-world research, enhancing this ability could be a valuable aid to successful practice. Our proposal for developing a repository of researchers' accounts of their experiences could be particularly fruitful in this regard. While this is useful, we feel that coping with uncertainty should remain a secondary aim as simply coping with uncertainty does little to support the reliability of assessment outcomes for those being assessed.

This paper is an example of sharing researcher's real-world experiences with the capacity to consent assessment. It outlines several practical challenges experienced during our doctoral research projects, the nature of these challenges, and potential solutions. In doing so, we are perhaps breaking with the convention of writing up research as though it proceeded flawlessly, but we feel that such an account is required to initiate a broader body of work. As such, we are attempting to spearhead a movement for the betterment of research conduct and researcher experiences. That said, it should be acknowledged that the experiences presented in this paper are a small sample drawn from three researchers. These examples solely serve to illustrate the potential for 
sharing researcher experiences. We do not suggest that they are representative of all researchers, though personal correspondence suggests that many others have experienced uncertainty. Instead, we wish to stimulate the publication of other experiences, be they similar or dissimilar. To this end, the systematic collation of a large sample of researcher experiences of the MCA warrants a future research project. Related journals appear to be an appropriate site for the development of a body of literature, although resulting work could also be collated into a unified digital resource available alongside the MCA. This could perhaps be located within the Social Care Institute for Excellence's MCA Forum (SCIE 2018), an online platform specialising in information regarding the MCA.

Finally, while this paper focusses on a single piece of legislation, belonging to England and Wales specifically, some of the core issues regarding mental capacity are somewhat internationally applicable. For example, international comparison reveals similar problems of varied practices when implementing capacity legislation (Fistein et al. 2009). In this respect, our suggestions speak to problems that are evident in the enactment of capacity legislation in other countries. However, there are considerable differences between the legislative approaches of some nations (Gray et al. 2010). Given the peculiarities of national legislation, the practical focus of a literature recounting researcher experiences would likely be heavily country-specific. It is the broader issue of the legitimacy of the concept of mental capacity that is a truly international concern, particularly in light of the UNCRPD (Bartlett 2012; Booth 2012; Gooding and O'Mahony 2016).

\section{Conclusion}

It is important to state that we are broadly supportive of the MCA and do not reject the 
underlying principle of mental capacity. ${ }^{4}$ This paper simply presents an argument for greater clarity regarding the MCA's research provisions. It suggests that this is achieved through more detailed guidance relating to various challenges that could occur, accompanied by the development of a body of work recounting researchers' experiences of using the MCA in practice. The paper kickstarts this effort through presenting the problems experienced by three researchers during their respective projects. Each of us felt confident in our knowledge of the MCA before starting our research, yet we each soon encountered unanticipated problems in practice. Study A struggled with the subjectivity of assessment, Study B experienced difficulty negotiating gatekeeper interpretation of capacity, and Study $\mathrm{C}$ found it hard to reconcile legal requirements with ethical and sensitivity considerations. These problems lead to uncertainties regarding the correct next actions to take. Despite varied degrees of prior training, each of us had to negotiate these emerging uncertainties as we encountered them, unsure of the legal and moral implications of our actions. While we did overcome challenges, we were caused unnecessary anxiety in the process of doing so.

It is unlikely that such anxieties are rare. While inevitable to an extent, they could likely be better moderated. We suggest that improved MCA guidance is needed to deal with uncertainties within the research provisions. We also suggest the development of a substantial literature documenting researchers' challenges and successes when implementing the MCA's research provisions. Such accounts may appear in articles such as this one, although their effectiveness could undoubtedly be maximised through

\footnotetext{
${ }^{4}$ See Booth 2012 and Gooding and O'Mahony 2016 for discussion of the legitimacy of mental capacity in legislation
} 
their compilation within an accessible, searchable resource alongside the MCA, perhaps hosted by the MCA Forum.

\section{Funding}

This work was supported by the Economic \& Social Research Council under Grant 1440363; Alzheimer's Society under Grant 17/IEC08/0003; and Economic \& Social Research Council under Grant 1014306.

\section{Declaration of Interests}

The authors declare no conflicting interests.

\section{References}

Banner, N. F. 2012. "Unreasonable reasons: normative judgements in the assessment of mental capacity." Journal of Evaluation in Clinical Practice, 18 (5): 1038-1044.

Bartlett, P. 2012. "The United Nations Convention on the Rights of Persons with Disabilities and Mental Health Law." The Modern Law Review 75 (5): 752-778.

Bernard, H. R. 1995. Research Methods in Anthropology: Qualitative and Quantitative Approaches. 2nd ed. London: Sage Publications.

Booth K. G. 2012. "Changing paradigms: mental capacity, legal capacity guardianship, and beyond." Columbia Human Rights Law Review, 44: 115-152.

Brown, R., P. Barber, and D. Martin. 2009. The Mental Capacity Act 2005: A Guide for Practice (Post-Qualifying Social Work Practice Series). 2nd ed. Exeter: Learning Matters.

Coffey, A., and P. Atkinson. 1996. Making Sense of Qualitative Data: Complementary Research Strategies. London: Sage Publications.

Department for Constitutional Affairs. 2007. Decision-Making Capacity Act 2005: Code of Practice. London: The Stationary Office.

Dobson, C. 2008. Conducting Research with People not Having the Capacity to Consent to their Participation: A Practical Guide for Researchers. Accessed 11 April 2018. http://www.ed.ac.uk/files/atoms/files/bps_guidelines_for_conducting_research_ with_people_not_having_capacity_to_consent.pdf. 
Fistein, E.C., Holland, A.J., Clare, I.C.H. and Gunn, M.J. 2009. “A comparison of mental health legislation from diverse Commonwealth jurisdictions." International Journal of Law and Psychiatry, 32 (3): 147-155.

Gooding, P. and O'Mahony, C. 2016. "Laws on unfitness to stand trial and the UN Convention on the Rights of Persons with Disabilities: comparing reform in England, Wales, Northern Ireland and Australia." International Journal of Law, Crime And Justice, 44: 122-145.

Gray, J.E., McSherry, B.M., O'Reilly, R.L. and Weller, P.J. 2010. “Australian and Canadian mental health Acts compared." Australian and New Zealand Journal of Psychiatry, 44 (12: 1126-1131.

Hotopf, M. 2005. “The Assessment of Decision-Making Capacity.” Clinical Medicine 5: $580-584$.

House of Lords. 2014. Mental Capacity Act 2005: Post-Legislative Scrutiny. Accessed 11 April 2018.

https://www.publications.parliament.uk/pa/ld201314/ldselect/ldmentalcap/139/1 39.pdf.

Iphofen, R. 2009. Ethical Decision Making in Social Research. Basingstoke: Palgrave Macmillan.

Law Commission. 2017. Decision-Making Capacity and Deprivation of Liberty. Law Com No 372. Accessed 11 April 2018. http://www.lawcom.gov.uk/app/uploads/2017/03/lc372_mental_capacity.pdf.

Mackenzie, R. and Watts, J. 2010. "Mind the gap: the Deprivation of Liberty Safeguards in the amended Mental Capacity Act 2005." Tizard Learning Disability Review, 15 (1): 51-55.

Manthorpe, J., Rapaport, J. and Stanley, N. 2008. "Expertise and experience: People with experiences of using services and carers' views of the Mental Capacity Act 2005.” British Journal of Social Work, 39 (5): 884-900.

Manthorpe, J., Samsi, K., Heath, H. and Charles, N. 2011. “"Early days': knowledge and use of the Mental Capacity Act 2005 by care home managers and staff." Dementia, 10 (3): 283-298.

Mental Capacity Act. 2005. Accessed 11 April 2018. http://www.legislation.gov.uk/ukpga/2005/9/pdfs/ukpga_20050009_en.pdf. National Decision-Making Capacity Forum. 2017. Research and Impaired DecisionMaking Capacity in Adults: Guidance for Researchers. Accessed 11 April 2018. 
https://www.healthandcareresearch.gov.wales/uploads/News/research_and_impa ired_mental_capacity_in_adults-guidance_for_researchers.pdf.

NICE. 2017. Draft Guideline: Decision-Making and Mental Capacity. Accessed 11 April 2018. https://www.nice.org.uk/guidance/gid-ng10009/documents/draftguideline.

Nordstrom, S. N. 2013. “Object-Interviews: Folding, Unfolding, and Refolding Perceptions of Objects." International Journal of Qualitative Methods 12: 236257.

Parker, J., B. Penhale, and D. Stanley. 2011. "Research Ethics Review: Social care and Social Science Research and the Decision-Making Capacity Act 2005." Ethics and Social Welfare 5 (4): 380-400.

SCIE. 2018. Mental Capacity Act Forum. Accessed 11 April 2018. https://www.scie.org.uk/mca-directory/forum/.

Sheather, J. 2006. “The Mental Capacity Act 2005.” Clinical Ethics, 1 (1) pp.33-36.

Wilson, E., Seymour, J.E. and Perkins, P. 2010. "Working with the Mental Capacity Act: findings from specialist palliative and neurological care settings." Palliative Medicine, 24 (4): 396-402. 